\section{(6) OPEN ACCESS}

\title{
Risk and radical uncertainty in HIV research
}

\author{
Caspar Hare
}

\section{Correspondence to \\ Professor C Hare, Department of Philosophy, MIT, Cambridge, MA 02139, USA; casparh@mit.edu}

Received 15 April 2016 Revised 22 August 2016 Accepted 29 August 2016 Published Online First 26 September 2016

\begin{abstract}
How much risk can we expose our research subjects to? There is a special challenge answering this question when the evidence on which we base our assessments of risk is fragmentary, conflicting or sparse. Such evidence does not support precise assignments of risk (eg, there is a $24.8 \%$ chance that this patient will develop AIDS in the next year if she participates in my study). At best it supports imprecise assignments of risk (eg, there is between a $5 \%$ and $35 \%$ chance that this patient will develop AIDS in the next year if she participates in my study). Here I discuss three approaches to evaluating risk when probability assignments are imprecise - an optimistic approach, a moderate approach and a pessimistic approach. I offer a practical reason to favour the pessimistic approach.
\end{abstract}

A central question in HIV research ethics is this: "Antiretroviral therapy for HIV works pretty well. HIV positive patients who interrupt it for the sake of participating in our research studies are often taking a risk. How much of a risk should we, the researchers, allow them to take?"

One reply: "We should allow them to take whatever risks they informedly consent to. They are autonomous adults. So long as we have told them all that we know about the possible costs and benefits associated with participating in the study, we should respect their decisions to participate".

Another reply: "Fully informing research subjects will sometimes involve presenting to them a complex picture that they are unqualified to assess. In these situations, particularly when the possibility of a 'cure' is salient to them, some subjects may want to take risks that we, the researchers, judge to be desperate. It is not okay to be complicit in desperate risk taking. It is doubly not okay to do that when we stand to benefit from the desperate risk taking. There is an upper bound to how much risk we should allow our subjects to take".

If we take the second reply seriously, then we have the job of specifying just what the upper bound is, and explaining how we tell whether it is being exceeded. Roughly speaking, this will be a matter of comparing the patients' chances if they participate in the study with their chances if they do not. Less roughly speaking, it will be a matter of comparing the prospect for the patient associated with participating in the study with the prospect for the patient associated with continuing with antiretroviral therapy. (Generally, the prospect for you associated with an option is the set of all things that might happen to you if you take it, weighted by the chances they will happen if you take it. So the prospect for the patient associated with continuing with antiretroviral therapy might be a $10 \%$ chance of living a further 50-60 immunocompromised years, a $20 \%$ chance of living a further 40-50 immunocompromised years..., etc). If the expected value of the prospect associated with participating (think of the expected value of the prospect as the sum of the values for the patient of each of the things that might happen, weighted by the probability that it will happen) is sufficiently close (what counts as sufficiently close?- that will be depend on just how desperate or self-sacrificial we want to allow our research subjects to be) to the expected value of the prospect associated with not participating, then we should allow participation. Otherwise we should not.

But there's a problem. Antiretroviral therapy for HIV is 30 years old. Millions of people have used it. We have an enormous amount of excellent statistical data on its effectiveness. On the basis of these data, we can often assign very precise numbers to our patients' chances if they continue with the therapy. But typically, we do not have data like this to go on when assessing our patients' chances if they participate in the study. How likely is it that a patient will develop AIDS within a year if he or she participates? We can look to the results of animal trials, the hunches of a group of prominent scientists, our understanding of viral mechanisms-and all that may be helpful, but it does not suggest a precise number.

Generally, in situations in which we have fragmentary, conflicting or sparse evidence for a proposition, philosophers like to say that it is rational to have imprecise levels of confidence in the proposition. Contrast, for example, the proposition that the next two coins I toss will both come up heads, with the proposition that fully autonomous vehicles will be legal throughout the city of Boston by the year 2036. How confident am I in the former? I am precisely $25 \%$ confident. How confident am in the latter? I have some grounds for confidence (Silicon Valley is marching ahead with the technology, the economic incentives for fully autonomous vehicles are considerable..., etc), and some grounds for scepticism (these technologies often take longer than one expects to mature, Boston is a challenging urban environment..., etc). Because my evidence is fragmentary, conflicting and sparse, it does not collectively license an attitude of $53.4688109353 . . . \%$ confidence, or any attitude so precise.

But to say that a level of confidence is imprecise is not to say that it cannot be represented by numbers; it is just to say that a single number will not do. I am more than 5\% confident that autonomous vehicles will be legal throughout Boston by 2016; I am less than 95\% confident of that. My 
confidence can be represented by a rough boundaried intervalfrom around $25 \%$ to around $45 \%$.

The problem is how to assess whether or not participation is too risky when our levels of confidence in the prospects associated with participation are imprecise. Three basic approaches spring to mind.

\section{The optimistic approach}

Take the most optimistic precisification of your imprecise levels of confidence in the prospects associated with participation. If participation is too risky, under that precisification, then do not allow it.

The moderate approach

Take the precisification at the mid-point of your imprecise levels of confidence in the prospects associated with participation. If participation is too risky, under that precisification, then do not allow it.

The pessimistic approach

Take the most pessimistic precisification of your imprecise levels of confidence in the prospects associated with participation. If participation is too risky, under that the precisification, then do not allow it.

Suppose my confidence that a patient will develop AIDS within a year if she participates in my research study is best represented by a rough boundaried interval from around 5\% to around 30\%. The optimistic approach would have me imagine my being given a large volume of statistical evidence that licenses precise 5\% confidence in her developing AIDS within a year. If and only if it would be okay for me then, with precise $5 \%$ confidence, to allow participation, then it is okay for me now, with imprecise 5-30\% confidence, to allow participation. The pessimistic approach would have me imagine my being given a large volume of statistical evidence that licenses precise $30 \%$ confidence in her developing AIDS within a year. If and only if it would be okay for me then, with precise 30\% confidence, to allow participation, then it is okay for me now, with imprecise 5$30 \%$ confidence, to allow participation. The moderate approach would split the difference.

Which approach is right? Philosophers who write about rationality tend to adopt a more optimistic approach to the rational permissibility of action. Their idea is that with imprecision in levels of confidence comes an expansion in the range of things that it is rationally permissible to do. ${ }^{1-4}$ If you have $40 \%$ confidence in a proposition and I offer you an even bet on it (if it is true I pay you a dollar, if it is false you pay me a dollar) then it is rationally permissible for you to refuse the bet, rationally impermissible for you to take it. But if you have imprecise $20-60 \%$ confidence in the same proposition, then it becomes both rationally permissible for you to refuse it (because it would be rationally permissible for you to refuse it if you were precisely $20 \%$ confident) and rationally permissible for you to take it (because it would be rationally permissible for you to take it if you were precisely $60 \%$ confident).

The philosophers have reasons for going this way. They see just three alternatives.

The lax theory of rational permissibility

It is rationally permissible for you to take an option if and only if it would be rationally permissible for you to take it under some precisification of your imprecise levels of confidence.

\section{The moderate theory of rational permissibility}

It is rationally permissible for you to take an option if and only if it would be rationally permissible for you to take it under the precisification at the mid-point of your imprecise levels of confidence.
The strict theory of rational permissibility

It is rationally permissible for you to take an option if and only if it would be rationally permissible for you to take it under every precisification of your imprecise levels of confidence.

They find the strict theory unsatisfactory because it says that very often there is nothing that it is rationally permissible to do. So, for example, when you have $20-60 \%$ confidence in a proposition, the strict theory says it is neither rationally permissible to accept my even bet on it nor rationally permissible to refuse my even bet on it. They find the moderate theory unsatisfactory because it says there is no practical difference between having imprecise $20-60 \%$ confidence in a proposition and having precise $40 \%$ confidence in a proposition. Surely there is a difference. So they are left with the lax theory.

These general considerations about rational permissibility might seem to tell in favour of the optimistic approach to enrolling research subjects. After all, enrolling research subjects is just a special case of rational action. And there are obvious further policy reasons to favour the optimistic approach. The bar for participation will be much lower. We will get more research subjects.

But here I want to suggest one less obvious policy reason to favour the pessimistic approach to enrolling research subjects. A peculiar feature of research studies is that, typically, they generate good statistical data on the prospects of their own subjects. Because of this, we can often reasonably expect that our attitudes, once the study is concluded, will be different and more precise than our attitudes now. The intervals representing our imprecise levels of confidence will in some way shift and contract. Just how will they shift and contract? Where our present confidence that the patient will develop AIDS within a year on this medication is $5-30 \%$, will it later be $2-4 \%$ ? $4-10 \%$ ? $20-$ 25\%? 25-40\%? 80-90\%? Typically, we do not know. Indeed, typically, we do not have precise levels of confidence about our future levels of confidence. But there is something we can say. Here are three propositions about our future levels of confidence in bad outcomes for our research subjects.

Rising lower bound

The lower bound of our future levels of confidence will exceed the lower bound of our present levels of confidence.

\section{Rising mid-point}

The mid-point of our future levels of confidence will exceed the mid-point of our present levels of confidence.

\section{Rising upper bound}

The upper bound of our future level of confidence will exceed the upper bound of our present levels of confidence.

Because we expect the intervals representing our imprecise levels of confidence to contract, we should be more confident in rising lower bound than in rising mid-point, and we should be more confident in rising mid-point than in rising upper bound. (Why? Note that, if the interval contracts, any shift that raises the mid-point also raises the lower bound, but some shifts that raise the lower bound do not raise the mid-point, and any shift that raises the upper bound raises the mid-point, but some shifts that raise the mid-point do not raise the upper bound.)

This means that, if we adopt the optimistic or moderate approach, we should be more confident that we are enrolling subjects in a study that will generate evidence in light of which it will be impermissible, by our own standards, to enrol them, than if we adopt the pessimistic approach. But it is bad, other things being equal, to enrol subjects in a study that generates evidence in light of which it is impermissible, by our 
own standards, to enrol them. What should we do, as this evidence begins to accumulate? Our options are effectively to ignore the accumulating evidence until the study is complete or to refuse to allow the now too risky subjects to participate further in the study. Neither of these options is good. Just as it is not okay to allow subjects to take risks that we consider desperate, so it is not okay to allow subjects to take risks that we know we would consider desperate, if we were not now wilfully ignoring evidence. And releasing research subjects mid-study is a waste of time and resources-better never to have enrolled them at all.

So we have a reason to be pessimistic in our assessment of risks to our research subjects. That will allow us to expect to keep them in our studies without exposing them to risks that are by our own lights unacceptable.
Funding This work was supported by the National Institute of Allergy and Infectious Diseases (NIAID) grants Nos 1 R01 Al114617-01A1 and 1 R56 Al114617-01.

Competing interests None declared.

Provenance and peer review Commissioned; externally peer reviewed.

Open Access This is an Open Access article distributed in accordance with the Creative Commons Attribution Non Commercial (CC BY-NC 4.0) license, which permits others to distribute, remix, adapt, build upon this work non-commercially, and license their derivative works on different terms, provided the original work is properly cited and the use is non-commercial. See: http://creativecommons.org/ licenses/by-nc/4.0/

\section{REFERENCES}

1 Levi I. The enterprise of knowledge. Cambridge, MA: MIT Press, 1980.

2 White R. Evidential symmetry and mushy credence. In: Gendler T, Hawthorne J, eds. Oxford studies in epistemology. Oxford: Oxford University Press, 2009:161-86.

3 Elga A. Subjective probabilities should be sharp. Philosophers' Imprint 2010;5.

4 Hare C. Take the sugar. Analysis 2010;70:237-47. 